\title{
Option Of The Method of Successive Approximations In Calculating The Epicenters Of Extreme And Emergency Situations
}

\author{
Kubaymurat Ismaylov, Professor, Mechanics of Deformable Rigid Body, Samarkand State Architectural \\ and Civil Engineering Institute, Samarkand, Uzbekistan, \\ Adylzhan Suleymanov, Professor of Tashkent State Technical University, Tashkent, Uzbekistan, \\ Sobirjob Toshev, Professor, Mechanics of Deformable Rigid Body, Samarkand State Architectural and Civil \\ Engineering Institute, Samarkand, Uzbekistan, \\ Sukhrob Ruziev, Assistant of the Department Technology of construction and its organization, Samarkand \\ State Architectural and Civil Engineering Institute, Samarkand, Uzbekistan.
}

\begin{abstract}
The mechanics of aspiration flows formation is considered in the paper as an additional safety mechanism. A solution to nonlinear differential equation describing the equilibrium of a closed cylindrical shell subject to axisymmetric radial pressure is obtained in the framework of strain theory of plasticity and on the basis of an option of the method of successive approximations for calculating the epicenters of emergency phenomena. It is assumed that the nonlinear components related to the ring strength of plastic zones are located near the shell edges of the surrounding operative environment.

The problem solutions are generalized for the case when the nonlinear components of the ring strength of the zone of emergency phenomena as a plastic zone are located near the shell of the operative headquarters created in an emergency. As a result of the studies, the plastic state of the shell between the linear state and the state of limit equilibrium of the zone of emergency is determined. This makes it possible to confine the center of extreme and emergency situations zone.

The test results conducted for various building structures are given. It is proposed to use the knowledge of aspiration flows strength as an additional safety mechanism, as an option of additional fire safety mechanism.
\end{abstract}

Keywords: aspiration coefficient mechanics; safety mechanism; combustion chamber; additional flow from the source; strain theory of plasticity; machines and mechanisms; axisymmetric strains; zone of emergency; epicenter.

Received: 04.12.2020 $\quad$ Accepted: 15.01.2021 $\quad$ Published: 07.02.2021

\section{Introduction}

Additional aspiration flows generated in the combustion zone are the indices of the combustion process rate, its direction and intensity. So, the study of the mechanics of aspiration flows generated during burning will provide additional necessary information.

\section{Statement of problem}

The need to study the mechanics of formation and risk reduction processes in emergency situations shows its relevance; this is a widely discussed scientific topic at many symposia and conferences on safety.

For the safety of human lives and health, it is necessary to take into account a number of probabilistic safety factors.

1. The first stage of emergency response can be described as a study of the protected system. From the very beginning, it is necessary to clearly determine how much time is available to solve the problems at this stage (this is especially necessary when we are dealing with a collapsing system). Here the determining factor is the maximum time for the development of the destructive process till the point " $S$ ", after which the destruction or collapse of the system can no longer be prevented (in other words, $S$ - is the point at which the restoration process is still possible). Based on the time available, it is possible to define the possible limit for the first stage [6-9].

The importance of the first stage is determined by the value of time spent on solving this problem. One of the possible ways to prolong this time is to invite the specialists and (or) setting specific tasks for people who have sufficient experience in solving them, since they are capable of:

firstly, accelerate the solution of problems;

secondly, quickly and reasonably determine the decisive course of action, based on prevailing conditions; 
thirdly, if necessary, to "sift" unnecessary (or temporarily unnecessary) subparagraphs of the first and subsequent stages. The time spent on inviting the specialists, obtaining information and making decisions can be designated as $t_{k}$ - the time that catalyzes the temporary "strain".

2. At the second stage of duration $t_{2}$ :

- the ways of distribution localization of the components that destroy the system are determined. In particular, these types of operations were carried out in Washington to localize the spread of outbreaks in connection with emergency situations and terrorists attacks;

- recommendations are being developed to maintain the strength of the system as a whole. For example, a set of preventive measures has been developed in the fire safety system, the purpose of which is to prevent fire in various spheres of industry; the Ministry of Emergency Situations has developed preventive measures that are applicable in wide areas of human life.

3. At the third stage of duration $t_{3}$ the following steps are conducted:

- operative intervention to localize the stability violation development;

- the installation (implementation) of an additional subsystem (the so-called "satellite") to increase the system reliability (or its necessary qualities, preferably without deterioration of the rest ones). However, the installation of an additional subsystem is often reduced to the implementation of a well-known principle - "if you want to buy something, you have to pay or sacrifice something". In practice, this is associated with the introduction of additional human and other reserves;

- re-arrangement of components (done less frequently), which allows to ultimately increase the system reliability and (or) stability;

- replacement or prompt removal, if necessary, of individual components that interfere with the stable existence of the system;

- establishing supervision of compliance with recommendations that contribute to increased reliability and stability;

- providing favorable conditions for the efforts that contribute to the stable functioning of the system.

\section{Mathematical mechanics of safety provision}

1. Time $t_{e m}$ available for the mechanics to study the processes of aspiration flows formation in the localization of the aftermath of emergency situations (ES) can be divided into three components:

$t_{e m}=t_{1}+t_{2}+t_{3}=\sum_{i=1}^{3} t_{i}$,

where $t_{e m}$ is the available time, that is, the time till the point " $\mathrm{Em}$ " (when it can be assumed that the aftermath are localized); $t_{i}$ is the time spent for each stage (where $i=1,2,3$ ); $t_{1}$ is the time to determine the operative situation; $t_{2}$ is the time for making a management decision; $t_{3}$ is the time for operative intervention $[12,17]$.

2. If to take into account that the three indicated time periods consist of the specific actions described above, the implementation of which also takes time, then these three time periods can be written in the following more specific form:

1) for the period of determining the operative situation $t_{1}=\sum_{j_{1}=1}^{N_{1}} t_{1 j_{1}}$, where $j_{1}$ may include the numbers of all possible measures of the 1st period;

2) for the period of making a managerial decision $t_{2}=\sum_{j_{2}=1}^{N_{2}} t_{2 j_{2}}$, where $j_{2}$ can cover the numbers of all possible measures of the 2nd period;

3) for the period of operative intervention $t_{3}=\sum_{j_{3}=1}^{N_{3}} t_{3 j_{3}}$, where $j_{3}$ can run through the numbers of all possible measures of the $3 \mathrm{rd}$ period $[6-10,14]$.

As a result of refining the components of each of the three time periods, we obtain a more detailed formula for the value of $t_{e m}$ : 


$$
t_{e m}=t_{1}+t_{2}+t_{3}=\sum_{j_{1}=1}^{N_{1}} t_{1 j_{1}}+t_{2}=\sum_{j_{2}=1}^{N_{2}} t_{2 j_{2}}+t_{3}=\sum_{j_{3}=1}^{N_{3}} t_{3 j_{3}},
$$

where $t_{1 j_{1}}$ - is the duration of measures to determine the operative situation, $j_{1}=1,2,3, \ldots, N_{1}, N_{1}$ - is the number of measures; $t_{2 j_{2}}$ - is the duration of managerial decision-making measures, $j_{2}=1,2,3, \ldots$, $N_{2}, N_{2}$ - is the number of these measures; $t_{3 j_{3}}$-is the duration of measures to implement the adopted managerial decision (direct operative intervention), $j_{3}=1,2,3, \ldots, N_{3}, N_{3}-$ is the number of these measures.

3. The sum (1) is confirmed if

$$
\begin{aligned}
& g\left(t_{1}\right)=0, \text { if } t_{1} \notin[a, b], \\
& \varphi\left(t_{1}\right)-0, \text { if } t_{3} \notin[c, d], \\
& g\left(t_{2}\right)=0, \text { if } t_{1} \in[b, c], \\
& \varphi\left(t_{3}\right)-0, \text { if } t_{3} \notin[c, d],
\end{aligned}
$$

where $g\left(t_{1}\right)$ - is the function to determine the operative situation; $g\left(t_{2}\right)$-is the function to make a managerial decision; $\varphi\left(t_{3}\right)$ - is a function for operative intervention. In system (2), parameters $a, b, c$ and $d$ describe preventive and tactical measures that can bring significant effectiveness to the activities and measures at the third stage. They can be realized at any of the three stages and even before the start of the first stage.

In a real situation, the durations of these three stages $t_{1}, i=1,2,3$, can vary significantly depending on how and when the manager will use the freed up time and use it at his disposal. If the manager effectively uses time $t_{1}$ to determine the operative situation, involving organizational and technical measures $t_{1 / 1}$ (for example, inviting consultants, more experienced employees, using dispatcher's meters, maps and plans, special embedded information), then the time duration shortens. This makes it possible to allocate more time for decision making, i.e., the time duration $t_{2}$ becomes longer. If the manager effectively used time $t_{2}$, optimally applying the measures $t_{2 / 2}$ then there is even more time left for the measures $t_{3 / 3}$ of time $t_{3}$ for operative intervention, for example, the rescue of the injured [11-12].

\section{An option of the method of successive approximations in calculating the epicenters of extreme and emergency situations}

When determining the decisive direction of the subsystems, all calculation methods developed so far can be divided into three groups: exact, approximate-analytical and numerical ones. Exact methods include the integral transform method and the separation method. However, these methods are used relatively rarely [13].

An option of the method of successive approximations is proposed for solving the basic nonlinear equation describing axisymmetric strains [15-16].

Integration of basic nonlinear equation describing the equilibrium of a closed cylindrical shell beyond the elastic limit under simple loading was obtained by A.A. Ilyushin. For radial load along the parallel it has the form:

$\frac{d^{4} w}{d x^{4}}+4 w=-\frac{4}{E} \frac{R}{h} \Delta N_{2}-\frac{6}{E} \frac{R}{h^{2}} \frac{d^{2} \Delta M_{1}}{d x^{2}}$

where: $w$ is the unknown function of the breaking point after the application of forces and measures; $R$ is the radius of the studied area; $h$ is the depth of the studied area; $E$ is the stress at the initial section $\sigma-\varepsilon ; x$ is the direction coordinate to the epicenter of the emergency zone, determined through the longitudinal coordinate $s$ by expression $x=\beta s ; \beta=\sqrt{3 / 2 R h} ; \Delta N_{2} ; \Delta M_{1}$ are the nonlinear components related to the ring tension strength in the emergency zone. The general solution of the basic equation (1) is presented in the form: 


$$
\begin{aligned}
& w=w(0) F_{1}(x)+\sqrt{\frac{2 R h}{3}} v(0) F_{2}(x)-\frac{6 R M_{1}(0)}{E h^{2}} F_{3}(x)- \\
& -\frac{6 R \sqrt{2 R h / 3}}{E h^{2}} Q_{1}(0) F_{4}(x)-\frac{6 R}{E h^{2}} Y(x)-\frac{4 R}{E h} Z(x) .
\end{aligned}
$$

Where $\quad Y(x)=\int_{0}^{x} F_{2}(x, \alpha) \Delta M_{1}(\alpha) d \alpha, \quad Z(x)=\int_{0}^{x} F_{4}(x, \alpha) \Delta N_{2}(\alpha) d \alpha$.

In formulas (4), (5), A.N. Krylov functions are denoted by $F_{1}, F_{2}, F_{3}, F_{4}$.

If to assume that functions $\Delta M_{1}(\alpha)$ and $\Delta N_{2}(\alpha)$ vary along the epicenter of the emergency zone located near the shell edges of the emergency zone, then functions (5) will be determined by the following expressions (Fig. 1) [13-14].

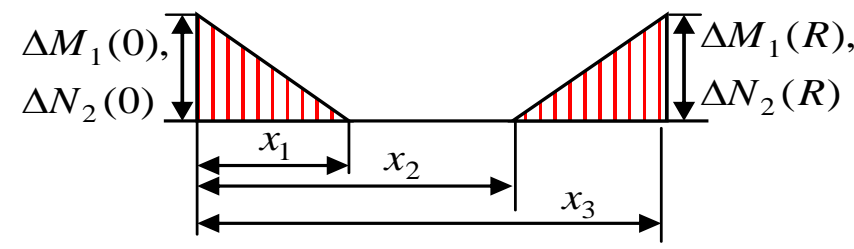

Fig.1 Diagrams of the problem statement for determining the epicenter of emergency zone system

a) the first section of the shell $0 \leq x \leq x_{1}$

$$
\begin{gathered}
Y(x)=\frac{\Delta M_{1}(0)}{x_{1}}\left[x_{1} F_{3}(x)-F_{4}(x)\right] ; \\
Z(x)=\frac{\Delta N_{2}(0)}{4 x_{1}}\left[x_{1}-x-x_{1} F_{1}(x)+F_{2}(x)\right]
\end{gathered}
$$

b) the second section of the shell $x_{1} \leq x \leq x_{2}$

$$
\begin{gathered}
Y(x)=\frac{\Delta M_{1}(0)}{x_{1}}\left[x_{1} F_{3}(x)-F_{4}(x)+F_{4}\left(x, x_{1}\right)\right] \\
Z(x)=\frac{\Delta N_{2}(0)}{4 x_{1}}\left[-x_{1} F_{1}(x)+F_{2}(x)-F_{2}\left(x, x_{1}\right)\right]
\end{gathered}
$$

c) the third section of the shell $x_{2} \leq x \leq x_{0}$

$$
\begin{aligned}
Y(x)= & \frac{\Delta M_{1}(0)}{x_{1}}\left[x_{1} F_{3}(x)-F_{4}(x)+F_{4}\left(x, x_{1}\right)\right] \\
& +\frac{\Delta M_{1}\left(x_{0}\right)}{x_{0}-x_{2}} F_{4}\left(x, x_{2}\right) ; \\
Z(x)= & \frac{\Delta N_{2}(0)}{4 x_{1}}\left[-x_{1} F_{1}(x)+F_{2}(x)-F_{2}\left(x, x_{1}\right)\right]+ \\
& +\frac{\Delta N_{2}\left(x_{0}\right)}{4\left(x_{0}-x_{2}\right)}\left[x-x_{2}-F_{2}\left(x, x_{2}\right)\right]
\end{aligned}
$$

Formulas (6) - (8) indicate that: $x_{0}$ - dimensionless length of the shell; $x_{1}$ - the length of the epicenter of the emergency zone located at the edge $x=0 ; x_{2}$ - the beginning of the zone epicenter at the edge $x=x_{0}$.

1) in the first section $0 \leq x \leq x_{1}$ 


$$
\begin{aligned}
& w(x)=-\frac{3 R}{4 E h^{2}}\left[q \sqrt{2 R h / 3} \varphi(x)-\Delta M_{1}(0) \frac{\Phi_{0}\left(x_{1}, x\right)}{x_{1}}-\right. \\
& \left.-\frac{h}{3} \Delta N_{2}(0) \frac{\Psi_{0}\left(x_{1}, x\right)}{x_{1}}+\frac{4}{3} h \Delta N_{2}(0)\left(1-\frac{x}{x_{1}}\right)\right] . \\
& \chi_{1}(x)=-\frac{9 R}{4 E h^{3}}[q \sqrt{2 R h / 3} \psi(x)- \\
& \left.-\Delta M_{1}(0) \frac{\Psi_{0}\left(x_{1}, x\right)}{x_{1}}+\frac{h}{3} \Delta N_{2}(0) \frac{\Phi_{0}\left(x_{1}, x\right)}{x_{1}}\right] . \\
& \Phi_{0}\left(x_{1}, x\right)=2 \varphi(x)-\varphi\left(x_{1}, x\right)- \\
& -\varphi\left(x_{1}\right) \theta(x)-\psi\left(x_{1}\right) \zeta(x) . \\
& \Psi_{0}\left(x_{1}, x\right)=2 \psi(x)-\psi\left(x_{1}, x\right)- \\
& -\psi\left(x_{1}\right) \theta(x)-\varphi\left(x_{1}\right) \zeta(x) .
\end{aligned}
$$

2) To obtain expressions of (9) - (11) type in the second section $\left(x>x_{1}\right)$, the following changes must be made: to omit the last term in expression (10); to replace functions $\varphi\left(x_{1}, x\right), \psi\left(x_{1}, x\right)$ by $\varphi\left(x, x_{1}\right), \psi\left(x, x_{1}\right)$, respectively, in formulas (10-11) [14,16].

Dependences (9) - (11) give a solution to the problem in initial approximation, when nonlinear components $\Delta M_{1}(x)$ and $\Delta N_{2}(x)$ vary along the emergency zone (Fig. 1).

In the second and successive approximations, this law is specified, that is, the indicated nonlinear components vary according to the law of a piecewise linear function for a specific emergency zone [1316].

A system of three nonlinear algebraic equations is derived to determine these three constants

$$
\begin{gathered}
\frac{3 R}{4 E h^{2}}\left[q \sqrt{\frac{2 R h}{3}}-\Delta M_{1}(0) \frac{\Phi_{0}\left(x_{1}, 0\right)}{x_{1}}-\right. \\
\left.-\Delta N_{2}(0) \frac{h \Psi_{0}\left(x_{1}, 0\right)}{3 x_{1}}+\frac{4 h}{3} \Delta N_{2}(0)\right]=\varepsilon_{i 0}(0) . \\
\varepsilon_{i 1}(0)=\sqrt{\varepsilon_{i 0}^{2}(0)+\left[h \chi_{1}(0)\right]^{2} / 3},
\end{gathered}
$$

For the final record of a nonlinear system of algebraic equations (10-12), it is necessary to express the quantities $\Delta M_{1}(0)$ and $\Delta N_{2}(0)$ through $\varepsilon_{i 0}(0)$ and $\varepsilon_{i 1}(0)$; this is done with formulas (10-11).

Thus, after such a transform, a system of three equations (13) - (14) is written relative to the following three unknowns: $\varepsilon_{i 0}(0)$ the minimum strain intensity in the initial section; $\varepsilon_{i 1}(0)$ the maximum strain intensity in the initial section and $x_{1}$ the zone length.

\section{Installation to detect aspiration flows arising from a fire hazard}

In the fire hazard study, the substances and materials that may be on people evacuation and rescue routes in emergency events were mainly tested as possible sources of hazardous factors (smoke formation coefficient, 


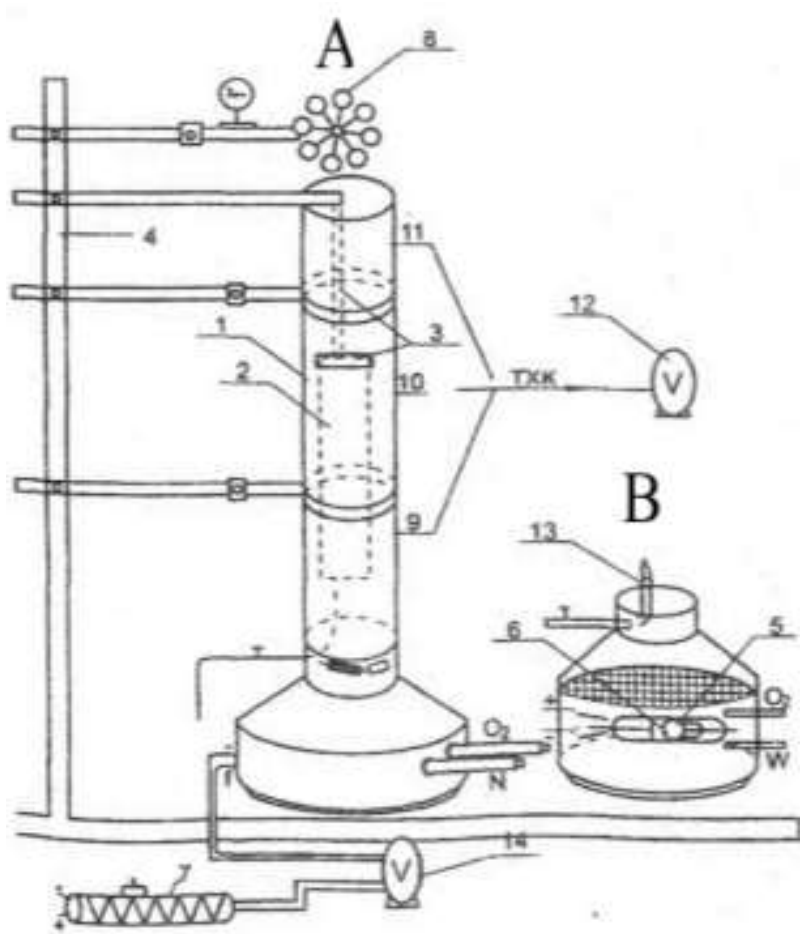

Figure 2. Installation for determining the aspiration coefficient in a fume hood: A) schematic view of the installation B) adjustment detail of the required parameters.

The detection of additional convective flow in a pipe makes it possible to determine the pattern in the combustion zone, depending on the rate of additional air flow. An additional convective flow arises due to the air inflow directly into combustion zone.

Knowing this, in turn, will make it possible to more effectively use the available capacities and measures in emergency situations.

The developed installation designed to determine additional aspiration flows in the combustion zone in a fire emergency is described below (Fig. 3.).

Such methods as the "Fire Pipe" [2]; "Ceramic pipe", "Oxygen index", state standards GOST 12,1.044-84 are designed to determine the degree of combustibility mainly in laboratory conditions, the method proposed by the authors allows assessing the circumstances directly during the fire. The installation for determining the aspiration coefficient in the combustion zone and around it presents a pipe made of technical porcelain (1) (pipe diameter is $50 \mathrm{~mm}$ ) fixed to a tripod with a special fastening of clamp type. An anemometer is located in the middle of the pipe; it displays readings on a potentiometer (5). Similar anemometer (6) is placed below the pipe 1. There is a burner (7) for laboratory testing.

Denote the number of revolutions of the lower and upper anemometer by $N_{1}$ and $N_{2}$, respectively. The aspiration coefficient is $K_{a s p}=N_{2} / N_{1}$. During the material burning, a decrease in pressure occurs directly around the burned material, due to the oxygen burning; as a result of pressure decrease, the air inflow occurs from the environment, the air flow is recorded by the lower anemometer.

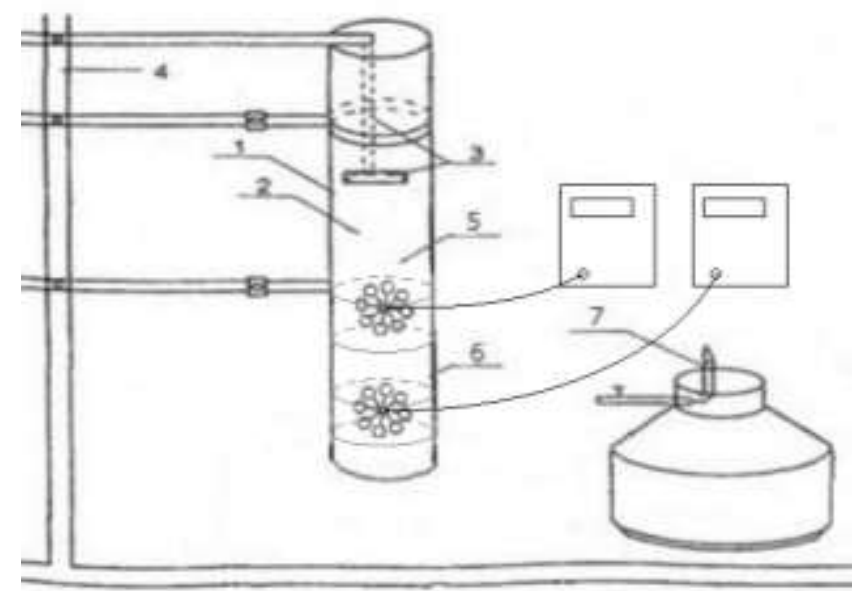


Schematic view of the installation

Figure 3 Installation for determining the aspiration coefficient in an open area

The physical meaning of the coefficient is determined by:

- the oxygen combustion ratio during material burning;

- the exhaustion ratio in the combustion volume;

Studies have confirmed that there is a definite relationship between the occurrence of aspiration flows during the combustion of test substances and materials and fire hazard.

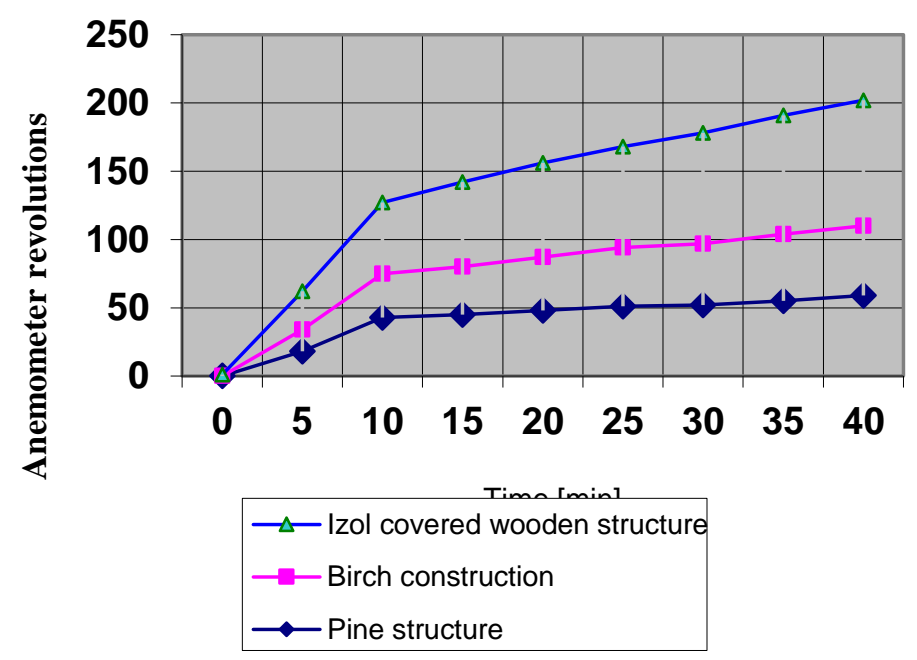

Fig. 4. Additional aspiration flows generated from the fire bed during structures burning

The test results with wood samples (pine, birch, a wooden structure covered with "Izol" combustible insulation material) conducted in field conditions are shown in Fig. 4.

The test results of building structures given in the graph showed that at the initial period, the aspiration flows in the combustion zone increase, obeying a linear equation $Y_{1}=19 x-19$.

A fracture occurs after the 10th second, and the description of the linear equation changes to the following one:

$Y_{2}=2,0571 x+33,629$.

Further, significant deviations from the indices of this equation no longer occur.

A sharp drop in coefficient before $x$ and an increase in the constant is explained not only by the formation of combustion products saturation in the test zone, but the basic role here is played by the fact that the characteristics of the combustion process go to the operating mode of the material being tested. The combustion process in the fire bed can be judged by the degree of activity and strength of aspiration flows. This allows calculating the strength and measures of reducing the risk of hazardous factors impact in advance.

\section{Conclusion}

The results presented in the study make it possible to determine the most likely source of fire bed at the moment. They allow calculating in advance the strength and measures of reducing the risk of hazardous factors impact.

\section{References}

1. Suleymanov A.A., Khasanov O.L. 2007. On science studying the development of security methodology. Actual problems of ensuring security in the Russian Federation. The First International Scientific and Practical Conference // Part 1. Yekaterinburg, - P. 53-55.

2. Suleymanov A. A., Khasanov 0. 2007. Security mechanism. Actual

3. problems of ensuring security in the Russian Federation // First International Scientific and Practical Conference. Yekaterinburg, Part 1. P. 3-5.

4. Ibragimov B. T., Suleymanov A. A. 2014. Adaptive systems in fireproof and seismic protection // Materials of a scientific and practical conference. Tashkent: IPB EMERCOM of the Republic of Uzbekistan, P. 45-47.

5. Suleimanov A. A., Ibragimov B. T. 2017. Research of Thermal and Mechanical Influence on FerroConcrete Designs with Damping Inserts // International Journal of Advanced Research in Science Engineering and Technology IJARSET. 4.9. P. 4595-4598.

6. Suleymanov A. A., Rustamy J. R., Akramov Zh. T. 2017. Theoretical and practical problems of 
ensuring safety in oil and gas industry // Science, Technology and Education. No. 10 (40). P. 2427.

7. Ibragimov B. T., Suleymanov A. A., Rustamy J. R. 2017. Entropy in assessing security processes // Materials of a scientific and practical conference. Tashkent: IPB Ministry of Internal Affairs of the Republic of Uzbekistan, P. 113-115.

8. Ibragimov B. T., Reimbaev R. S. 2016. On the issue of ensuring the safety of explosive industrial facilities located in the earthquake zone // Ibid. P. 56-58.

9. Majidov I.U., Ibragimov B.T., Suleimanov A.A. 2017. Protection in assessment of seismic hazard and safety processes // Scientific and Technical Journal of the Fergana Polytechnical Institute "Izvestiya FerPI". No. 1. P. 56-57.

10. Suleymanov A. A., Kurbanbaev Sh. E., Ibragimov B.T. et al. 2017. Energy value of the probability of safety ensuring from spontaneous evolution of a system // Scientific-Technical Journal of Tashkent Architecture and Construction Institute "Architecture and Design". No. 3-4. P. 108-111.

11. Yaskevich M.V., Suleymanov A.A., Tamanova V.V. 2018. A universal time vector for rescuing victims as a result of co-directed impact of seismic fire hazard // In collection of articles of students, masters, graduate students, young scientists and teachers "Development of modern science: theoretical and applied aspects." Issue 26. Perm, P. 58-59.

12. Rasul-Zade D. A., Suleymanov A. A., Budovkina A. A. 2018. Efficiency of advisory committees to reduce the risks probability of unit actions in emergency situations in oil and gas industry // New Information Technologies in Science: Collection of Articles of International Scientific -practical conference. Chelyabinsk, P. 17-19.

13. Vlasov V.Z. 1949. General theory of shells and its applications in technology. State Publishing House of Technical Literature, - Moscow-Leningrad.-784 p.

14. Ilyushin A.A. 1948. Plasticity. State publishing house of technical literature, - Moscow-Leningrad.$376 \mathrm{p}$.

15. Ismayilov K. 2003. Stability of compressed rods, plates and shells beyond the elasticity limit. FAN. - Samarkand.-280 p.

16. Suleymanov A.A., Gafforia M.A. 2018. Probability theory in risk assessment in emergency situations in oil and gas industry. Abstracts of the student scientific conference "Oil and Gas 2018", Branch of the Russian State University of Oil and Gas (NRU) named after I.M. Gubkin in Tashkent, Tashkent. - P. 75.

17. Suleymanov A.A. 2019. Measurement of aspiration flows generated during fires. Handbook. Engineering Journal with the application. M.: No 2 (263). - P. 42-48.

18. Fayziyev Shokhrud Farmonovich Medical law and features of legal relations arising in the provision of medical services. International journal of pharmaceutical research Volume 11, Issue 3, July - Sept, $2019 \quad$ P. $1197-1200$ doi:10.31838/ijpr/2019.11.03.088 http://www.ijpronline.com/ViewArticleDetail.aspx?ID=11016

19. Bryanskaya Elena, Fayziev Shokhrud, Altunina Anna, Matiukha Alena Topical Issues of an Expert Report in the Process of Proving in a Criminal Examination. International Journal of Engineering and Advanced Technology (IJEAT) ISSN: 2249 - 8958, Volume-9 Issue-1, October 2019 5345-5349 DOI: $\quad 10.35940 /$ ijeat.A2946.109119 https://www.ijeat.org/wpcontent/uploads/papers/v9i1/A2946109119.pdf

20. Fayziev Shokhrud (2019) Legal Aspects of Transplantology in the Republic of Uzbekistan. Systematic Reviews in Pharmacy, ISSN: 0976-2779, Vol: 10, Issue: 2, Page: 44-47 doi:10.5530/srp.2019.2.08 1575419211.pdf?1586863081

http://www.sysrevpharm.org//fulltext/196-

21. Tulaganova, G. Some issues of observance of international legal norms of fight against legalization of criminal incomes in the Republic of Uzbekistan Journal of Advanced Research in Dynamical and Control Systems 12(2 Special Issue), c. 143-155

22. Bazarova D. Some problems of counteracting crimes related to laundering of illegal proceeds in Uzbekistan Journal of Advanced Research in Dynamical and Control Systems. Volume 11, Issue 7, 2019, Pages 873-885 